\title{
A new genus and three new species of the family Theridiidae (Arachnida: Araneae) from North Borneo
}

\author{
Hajime Yoshida \\ 7-16, Kagota 2 Chome, Yamagata-shi, Yamagata, 990-2484 Japan \\ E-mail: araneae@mb.infoweb.ne.jp
}

\begin{abstract}
A new genus, Deelemanella, and three new species, D. borneo, Molione christae and M. kinabalu, belonging to the family Theridiidae are described from North Borneo.
\end{abstract}

Key words - Deelemanella, Molione, new genus, new species, Theridiidae, North Borneo

Through the kindness of Dr. Christa L. DeelemanReinhold, Ossendrecht, the Netherlands, I had an opportunity to study some theridiid spiders collected from North Borneo. In this paper, I wish to describe a new genus and three new species.

One of the three species is a member of the new genus Deelemanella belonging to Argyrodinae, and the other two are member of the known genus Molione Thorell 1892 of the subfamily Theridiinae. Deelemanella is characterized by male carapace with a dorso-anterior projection, and flat abdomen with some pairs of dorsal projections. Molione, under which three species has been recorded, is characterized by abdomen with extremely sclerotized epigastric area and a sclerotized ring around spinnerets; although three known species have dorsal spine-like projections on the abdomen, the present two new species lack such projections.

All the specimens used in this paper are deposited in the collection of the Department of Entomology, National Museum of Natural History Naturalis, Leiden, the Netherlands.

For the descriptions, following abbreviations are used in this paper: ALE, anterior lateral eye; AME, anterior median eye; AME-ALE, between AME and ALE; AME-AME, between AMEs; AW, anterior width; L, length; MOA, median ocular area; PLE, posterior lateral eye; PME, posterior median eye; PME-PLE, between PME and PLE; PME-PME, between PMEs; PW, posterior width.

\section{Deelemanella new genus}

Diagnosis. The present new genus resembles Argyrodes Simon 1864, but is distinguished from the latter by male carapace with only one projection intervening median eyes and flat abdomen with many dorsal projections.

This genus consists of only the type species and belongs to the subfamily Argyrodinae, which formerly consisted of four genera, Spheropistha Yaginuma 1957, Argyrodes Simon 1864, Rhomphaea L. Koch 1872 and Ariamnes Thorell 1869 (Yoshida 2001, 2003). Exline \& Levi (1962) treated Rhomphaea and Ariamnes as species groups of Argyrodes (s. lat.) respectively, and moreover divided Argyrodes (s. str.) into four species groups. This new genus does not belong to any of the species groups designated by them.

Description. Male carapace with a dorso-anterior projection intervening median eyes. Abdomen flat and oblong, with paired dorsal nipple-like projections. Epigynum swollen semi-globularly with a median opening. Male palpus with conductor membranous and long, and a large sclerite between conductor and cymbium.

Etymology. The generic name is after Dr. Christa L. Deeleman-Reinhold, and is feminine in gender.

Type species. Deelemanella borneo new species.

\section{Deelemanella borneo new species}

(Figs. 1-5)

Diagnosis. The present new species is an only member of this genus. The characters of abdomen and male carapace are not seen in the known species of the genus Argyrodes.

Description. Carapace oval and convex; both posterolateral edges bent backwards; a small projection present between AMEs in female; a dorso-anterior large projection present among median eyes in male; male clypeus projecting and truncated, with long hairs near AME (Figs. 1-2). Leg formula 1,4,2,3. Abdomen flat and oblong, with paired dorsal nipple-like projections (Figs. 1-2).

Female genitalia as shown in Figs. 4-5: epigynum swollen semi-globularly, with a median opening; ducts not so long.

Male palpus as shown in Fig. 3: conductor membranous and long, basally thin and distally thick; embolus with large 


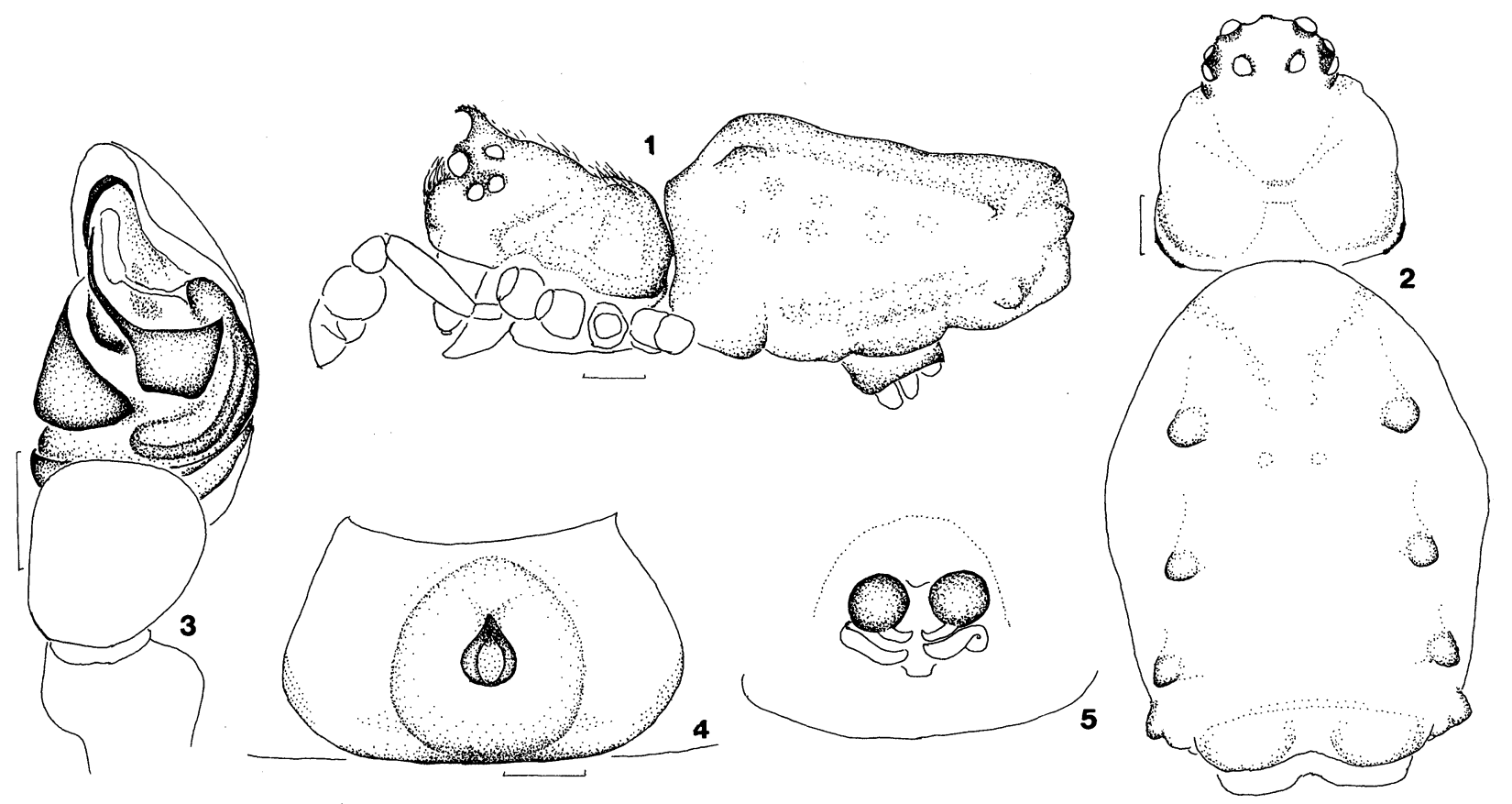

Figs. 1-5. Deelemanella borneo new species, ㅇ paratype and $\mathrm{o}^{\top}$ holotype -1 , male, lateral view; 2 , female carapace and abdomen, dorsal view; 3, male palpus, ventral view; 4, epigynum, ventral view; 5, female internal genitalia, dorsal view. Scales: $0.2 \mathrm{~mm}(1-2)$ and $0.1 \mathrm{~mm}$ $(3-5)$.

base, the tip long and pointed; a large sclerite present between conductor and cymbium.

Coloration (Figs. 1-2). Cephalothorax brown. Eyes on dark bases. Abdomen grayish brown with dusky flecks and dorsally with two pairs of brown disks.

Measurements (in mm, + paratype/ $/ \sigma^{\top}$ holotype). Body length 2.76/2.18. Carapace length 0.97/0.92; width $0.84 / 0.74$. Abdomen length $1.92 / 1.45$; width $1.21 / 0.74$; height 1.53/0.92. First leg: femur 1.24/1.13; patella and tibia 1.21/1.21; metatarsus $0.63 / 0.63$; tarsus $0.55 / 0.55$. Second patella and tibia $0.82 / 0.76$; third patella and tibia $0.55 / 0.47$; fourth patella and tibia 0.97/0.79. AME 0.12/0.12; ALE 0.08/0.07; PME 0.08/0.07; PLE 0.08/0.07. AME-AME $0.13 / 0.13$; AME-ALE 0.03/0.03; PME-PME 0.12/0.13; PME-PLE 0.08/0.07. MOA, AW 0.32/0.32; PW 0.24/0.26; L $0.24 / 0.18$.

Variations. Body length, $2.7-3.4 \mathrm{~mm}$ in female, $2.2-2.3$ $\mathrm{mm}$ in male.

Type series. Holotype: $\delta^{\lambda}$, and paratypes: $2{ }^{2} 1 \delta^{\lambda}, \mathrm{N}$. BORNEO, Mt. Kinabalu N. P., Poring Hot Springs, $6^{\circ} 2^{\prime} \mathrm{N}$ $116^{\circ} 50^{\prime} \mathrm{E}$, primary forest $500^{-} 700 \mathrm{~m}$, day-fogging canopy tree 52 Aporusa species (Euphorbiaceae), 19-II-1996 to 26III-1996, leg. A. Floren, ex coll. C. L. Deeleman 2000-704.

Distribution. North Borneo.

Etymology. The specific name is a noun in apposition after Borneo.

\section{Molione Thorell 1892}

Molione Thorell 1892, p. 216-Levi \& Levi 1962, p. 48; Wunderlich 1994, p. 569.

Note. Three species have been described under this genus. M. triacantha Thorell 1892 and M. trispinosa (O. Pickard-Cambridge 1873) have three dorsal spine-like projections on abdomen, and M. uniacantha Wunderlich 1995 has one dorsal projection. Two new species described in this paper has no dorsal projections. Abdomen with extremely sclerotized epigastric area and a sclerotized ring around spinnerets. Cymbium of male palpus with a hooded paracymbium. This genus belongs to the subfamily Theridiinae.

Though two new species lack dorsal projections on abdomen, extremely sclerotized epigastric area and a sclerotized ring around spinnerets clearly show that they belong to Molione.

Type species. Molione triacantha Thorell 1892.

Molione christae new species

(Figs. 6-9, 14-15)

Diagnosis. This new species is easily distinguished from the other three known species of Molione by abdomen without dorsal spine-like projections. This species most 

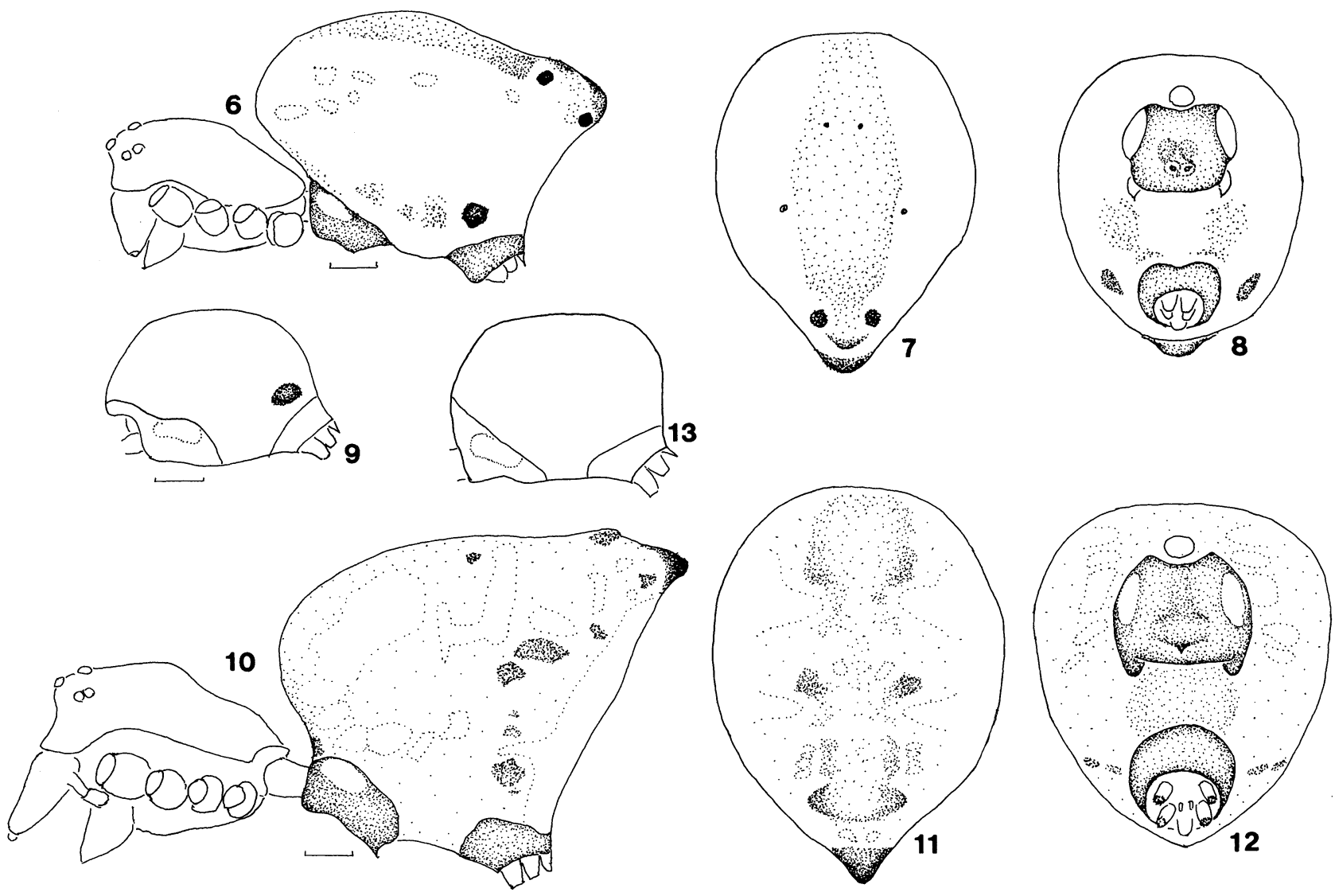

Figs. 6-13. Molione christae new species (6-9) and M. kinabalu new species (10-13), ㅇ holotype and $\sigma^{\lambda}$ paratype $-6,10$, female, lateral view; $7-8,11-12$, female abdomen, dorsal $(7,11)$ and ventral $(8,12)$ view; 9,13 , male abdomen, lateral view. Scales: $0.2 \mathrm{~mm}$.

resembles M. kinabalu new species, but is distinguished from the latter by male palpus with long conductor and female internal genitalia with short ducts. Body length of this species (female $1.6-2.2 \mathrm{~mm}$, male $1.5-1.8 \mathrm{~mm}$ ) is smaller than that of M. kinabalu (female 2.3-3.0 mm, male 2.0-2.1 $\mathrm{mm}$ ). Colorations of both species are also different.

Description. Carapace oval. Leg formula 1,4,2,3. Abdomen oval, posterior end projecting beyond spinnerets in female, not projecting in male; epigastric area, a ring around spinnerets and posterior projection sclerotized in female (Figs. 6-8), epigastric area and anterior part around pedicel forming a large sclerotized plate in male (Fig. 9). Pedicel sclerotized.

Female genitalia as shown in Figs. 8, 14: epigynum with a pair of small depressions, in which two openings situated in them; seminal receptacles globular; ducts short.

Male palpus as shown in Fig. 15: conductor long and membranous.

Coloration (Figs. 6-9). Carapace dusky brown, darker in head region. Chelicerae, maxillae and labium brown. Legs brown; first femora and tibiae with dusky flecks. Abdomen grayish brown with black or dusky flecks and small whitish pigments; sclerotized areas brown; posterior end with two pairs of black points; both lateral sides of spinnerets each with one large black points; dorsum with a median wide dusky fleck.

Measurements (in mm, 우 holotype/ð paratype). Body length 2.18/1.84. Carapace length $0.79 / 0.92$; width $0.68 / 0.61$. Abdomen length $1.50 / 1.00$; width $1.13 / 0.71$; height $1.13 / 0.97$. First leg: femur 1.05/1.16; patella and tibia $1.05 / 1.11$; metatarsus $0.63 / 0.79$; tarsus $0.32 / 0.37$. Second patella and tibia $0.71 / 0.82$; third patella and tibia $0.53 / 0.61$; fourth patella and tibia 0.87/0.92. AME 0.07/0.08; ALE 0.07/0.07; PME 0.07/0.08; PLE 0.05/0.07. AME-AME 0.07/0.07; AME-ALE 0.03/0.02; PME-PME 0.05/0.03; PME-PLE 0.05/0.07. MOA, AW 0.18/0.21; PW 0.18/0.18; L 0.13/0.13.

Variations. Body length, 1.6-2.2 mm in female, $1.5^{-1.8}$ $\mathrm{mm}$ in male.

Note. This species is seen only in the primary forest of fogging canopy Aporusa species (Euphorbiaceae), (Deeleman-Reinhold in personal communication).

Type series. Holotype: 우, and paratypes: 2 우 $2{ }^{\lambda}, 1$ 우 juvenile, N. BORNEO, Mt. Kinabalu N. P., Poring Hot Springs, $6^{\circ} 2^{\prime} \mathrm{N} 116^{\circ} 50^{\prime} \mathrm{E}$, primary forest $500^{-} 700 \mathrm{~m}$, fogging canopy Aporusa species (Euphorbiaceae), 19-II-1996 to 26-III1996, leg. A. Floren, ex coll. C. L. Deeleman 2000-704.

Distribution. North Borneo.

Etymology. The specific name is after Dr. Christa L. Deeleman-Reinhold. 
Molione kinabalu new species

(Figs. 10-13, 16-19)

Diagnosis. This species is easily distinguished from the other known species of Molione by the abdomen without dorsal projections. This species resembles $M$. christae new species. The discriminating characters are given under the diagnosis of $M$. christae.

Description. Overall external characteristics are similar to those of $M$. christae new species (Figs. 10-13). Female genitalia as shown in Figs. 12 and 16: epigynum with a small scapus, below which openings situated; seminal receptacles globular; ducts thin and long. Male palpus as shown in Figs. 17-19: embolus thick, the tip distally pointed; conductor membranous, the tip rounded; cymbium with hooded paracymbium.

Coloration (Figs. 10-13). Cephalothorax dark brown.
Abdomen grayish brown with large white pigments and black flecks; sclerotized areas brown.

Measurements (in $\mathrm{mm}$, 우 holotype/ $\sigma^{\Uparrow}$ paratype). Body length 2.95/2.08. Carapace length 1.24/1.00; width 0.74/0.71. Abdomen length 1.71/0.95; width 1.29/0.82; height 1.58/0.82. First leg: femur 1.03/0.97; patella and tibia 0.97/1.00; metatarsus $0.55 / 0.66$; tarsus $0.34 / 0.37$. Second patella and tibia $0.74 / 0.74$; third patella and tibia $0.61 / 0.58$; fourth patella and tibia 0.84/0.82. AME 0.09/0.09; ALE 0.07/0.08; PME 0.07/0.08; PLE 0.07/0.05. AME-AME 0.08/0.07; AME-ALE 0.03/0.03; PME-PME 0.07/0.05; PME-PLE 0.09/0.09. MOA, AW 0.24/0.24; PW $0.21 / 0.17$; L 0.14/0.16.

Variations. Body length, 2.3-3.0 $\mathrm{mm}$ in female, 2.0-2.1 $\mathrm{mm}$ in male.

Note. This species is seen in 40 years old secondary forest of fogging canopy Vinex pinnata (Verbenaceae) (Deeleman-Reinhold in personal communication).
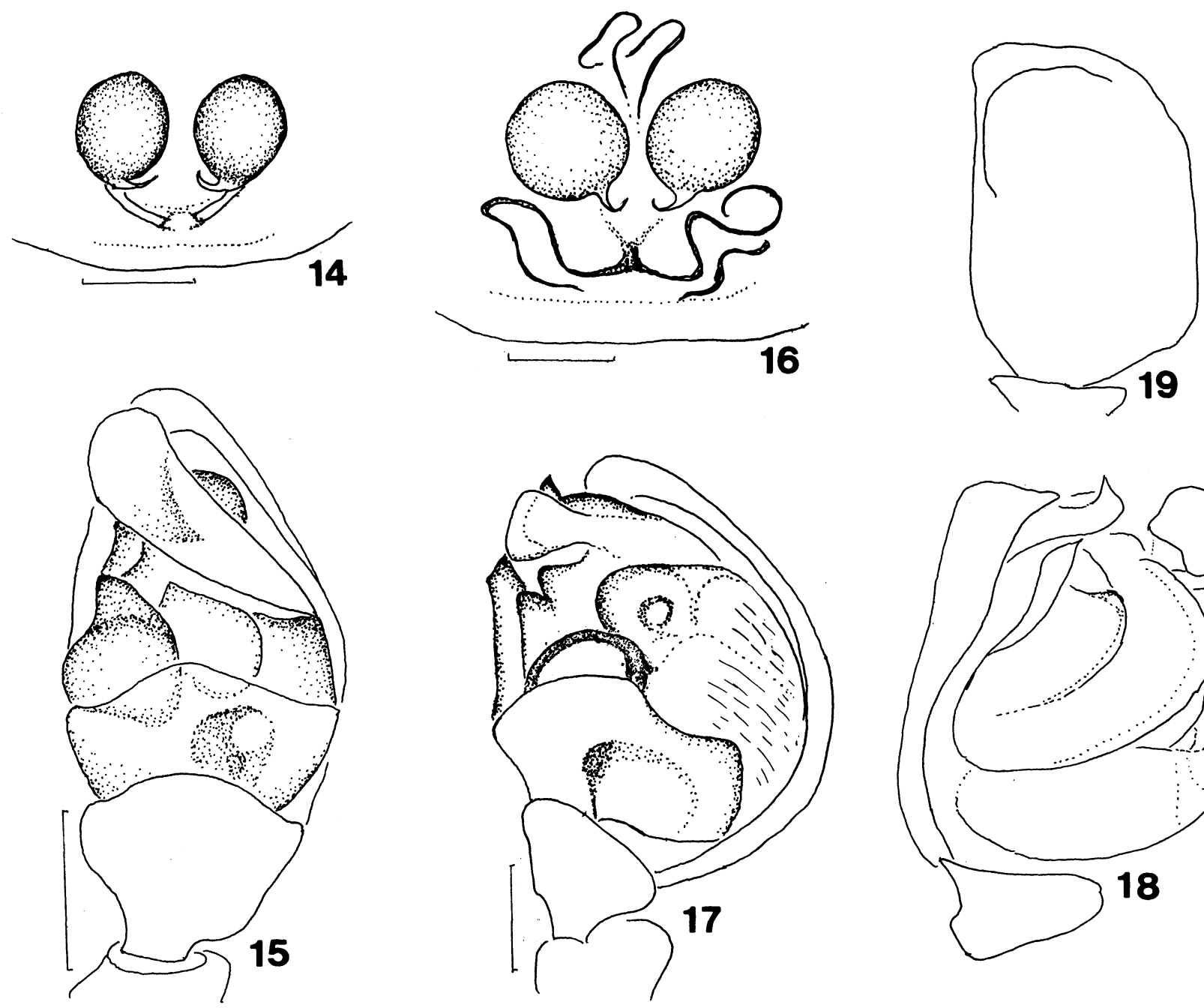

Figs. 14-19. Molione christae new species (14-15) and M. kinabalu new species (16-19), ㅇ holotype and $\delta^{\lambda}$ paratype - 14, 16, female internal genitalia, dorsal view; 15, 17-18, male left palpus, ventral (15), retrolateral (17) and prolateral (18, expanded) view; 19, cymbium of male left palpus, ventral view (bulb excluded). Scales: $0.1 \mathrm{~mm}$. 
Type series. Holotype: 우, and paratypes: 5 우 $30^{\lambda}, \mathrm{N}$. BORNEO, Mt. Kinabalu N. P., Poring Hot Springs, $6^{\circ} 5^{\prime} \mathrm{N}$ $116^{\circ} 50^{\prime} \mathrm{E}, 40$ years old secondary forest $500-700 \mathrm{~m}$, fogging canopy Vinex pinnata (Verbenaceae), 5 to 14-III-1997, leg. A. Floren, ex coll. C. L. Deeleman 2000-704.

Distribution. North Borneo.

Etymology. The specific name is a noun in apposition after Mt. Kinabalu.

\section{Acknowledgments}

I wish to express my sincere thanks to Dr. Christa L. DeelemanReinhold, Ossendrecht, the Netherlands, and Dr. Erik J. van Nieukerken, National Museum of Natural History Naturalis, Leiden, the Netherlands, for loaning many specimens used in this paper.

\section{References}

Exline, H. \& Levi, H. W. 1962. American spiders of the genus Argyrodes (Araneae, Theridiidae). Bull. Mus. Comp. Zool., 127: 75
$-204,15$ pls.

Levi, H. W. \& Levi, L. R. 1962. The genera of the spider family Theridiidae. Bull. Mus. Comp. Zool., 127: 3-71, 14 pls.

Pickard-Cambridge, O. 1873. On some new genera and species of Araneidea. Proc. Zool. Soc. London, 1873: 112-129, pls. XII-XIV.

Thorell, T. 1892. Studi sui Ragni Malesi e Papuani. Part IV, 2. Ann. Mus. Civ. Stor. Nat. Genova, 31: 1-490.

Wunderlich, J. 1994. Beschreibung bisher unbekannter Spinnenarten und -Gattungen aus Malaysia und Indonesien (Arachnida: Araneae: Oonopidae, Tetrablemmidae, Telemidae, Pholcidae, Linyphidae, Nesticidae, Theridiidae und Dictynidae). Beitr. Araneol., 4: 559579.

Yoshida, H. 2001. The genus Rhomphaea (Araneae: Theridiidae) from Japan, with notes on the subfamily Argyrodinae. Acta Arachnol., 50: 183-192.

Yoshida, H. 2003. The Spider Family Theridiidae (Arachnida: Araneae) from Japan. Arachnological Society of Japan, Osaka. 224 pp. (In Japanese)

Received September 30, 2003 / Accepted October 27, 2003 


\section{Acta Arachnologica Vol. 52, No. 2 掲載論文の和文要旨}

キクヅキコモリグモから出現した糸片虫科線虫（pp. 77-78） 飯田博之 $・$ 長谷川英男 ${ }^{2}{ }^{1}$ T 606-8502 京都市左京区北白川追 分町 京都大学大学院農学研究科昆虫生態学研究室; ${ }^{2}$ T $879-$ 5593 大分県大分郡挾間町医大ヶ丘 1 丁目 1 番地 大分医科大学 感染分子病態制御研究室)

京都市内の水田で採集したキクッ゙キコモリグモPardosa pseudoannulata 飼育していたところ，12 頭のクモから計 22 頭の糸片虫科線虫が出てきた。宿主は全て雌成体だった。 ほと んぞの場合，1頭のクモに寄生していた線虫は 1 頭だった。 し かし， 1 頭のクモに 2 頭および 10 頭の線虫が寄生していた場 合もそれぞれ 1 例ずつあった。 また 12 頭の宿主のうち，線虫 が出た後む生きていた個体は 2 頭だった，線虫の体長は 14 $159 \mathrm{~mm}$ であった. Poinar (1977，1979，1986）に基づき線虫の 同定を試みたところ，その形態的特徵から Mermis, Octomermis, Orthomermis, Allomermis, Pheromermis, Eumermis, Phreatomermis, Hydromermis, Quadrimermis, Aranimermis 属ではないことは判明 したが，幼体であったのでこれ以上同定することはできなかっ た. 寄生率については, 採集個体のおおよその数から判断する と，1〜10\%の範囲内であると推測できた，世界では 40 種以上 のクモ類から糸片虫科線虫が見つかっているが，キクッ゙キコモ リグモからの報告例はまだない. 糸片線虫科の線虫は幼体のス テージで寄生している（Hyman 1951）。したがって, 宿主から 出た直後はまだ幼体であるため種の同定はできない、今後, 線 虫の種を同定するためには, Poinar（1986）のように宿主から 出た線虫を成虫にまで育てる必要があるだろう.

Oxyptila atomaria（クモ目，カニグモ科）の日本からの発見 (pp. 79-81)

小野展嗣 ${ }^{1}$ ・松田まゆみ ${ }^{2}\left({ }^{1}\right.$ 个169-0073 東京都新宿区百人町 323-1 国立科学博物館動物研究部内 ; ${ }^{2}$ T $080-1403$ 北海道河東 郡上士幌町糠平北区 25)

土㙵性のカニグモ科の 1 種 Oxyptila atomaria (Panzer 1801) (アトムオチバカニグモ一新称一) を，北海道において主にピッ トホールトラップを用いて採集された標本に基づいて日本から 初めて記録し, 両性の生殖器を図示した。本種はヨーロッパか ら, シベリア, 韓国, 日本とユーラシア大陸北部に広く分布す るが，同大陸東部では此較的稀であると思われる.

\section{北海道産のカイゾクコモリグモ属（クモ目：コモリグモ科）の 1 新種 (pp. 83-84)}

田中穂積（干661-8520 兵庫県尼崎市南塚口町 7-29-1 園田学 園女子大学生物学研究室)

北海道天塩郡サロベッ原野, 苫小牧市弁天沼やウトナイ湖等 の湿原より得られたコモリグモ科カイゾクコモリグモ属の 1 新 種をPirata hokkaidensis ミズベコモリグモ（新称）と命名して 記載した。本種は中国に分布する，Pirata serrulatus Song \&
Wang 1984 に近似するが, 雄触肢中部把持器基部の突起がない ことによって区別できる.

北ボルネオ産ヒメグモ科（クモ綱：クモ目）の 1 新属および 3 新種 (pp. 85-89)

吉田 哉（†990-2484 山形市篭田 2 丁目 7 番 16 号）

北ボルネオから 1 新属 Deelemanella および 3 新種 D. borneo, Molione christae, M. kinabalu を記載した. Deelemanella (イソ ウロウグモ亜科）は雄の背甲の頭部に 1 つの突起があり，腹部 が扁平で背面に数対の突起があることにより他の属から区別で きる. Molione (ヒメグモ亜科) はこれまで腹部背面に刺状の 突起がある 3 種が知られているが，本稿で記載した 2 種は腹部 背面に突起がない.

アジア産トゲオオザトゥムシ亜科数種の正体とシノニム（ザト ウムシ目, マザトウムシ科) (pp. 91-102)

Wojciech Staręga (Instytut Biologii Akademii Podlaskiej, Poland) 多数の夕イプ標本と原記載にもとづきトゲオオザトウムシ亜 科（Opilioninae）の分類学的改訂をおこなった。いくつかの転 属と多数のシノニムが含まれる. 本亜科は次の属からなる： Bidentolophus, Egaenus, Himalphalangium, Homolophus, Opilio, Scleropilio. ほとんどの属と種は中央アジアと東アジアに分布 し，アジア西部とヨーロッパには数種が到達しているのみであ る.次の種については転属をおこなった：Egaenus pakistanus (Roewer), Himalphalangium spinulatum (Roewer)（=ゴホント ゲザトウムシ), Homolophus serrulatus (Karsch), Bidentolophus adungius (Roewer), Homolophus consputus (Simon), H. nigridorsus (Caporiacco), H. turcicus (Roewer)（最後の 3 種は やや不明確), H. martensi (Staręga) (以上, 転属後の学名). 新しくシノニムとなったのは次のとおり (等号の前が新参シ) 二ム) : Egaenus carpaticus Avram = E. convexus (C. L. Koch), Euphalangium afganum Roewer $と$ E. chitralense Roewer $=$ Egaenus kashmiricus Caporiacco, Opilio nigerrimus Schenkel = Himalphalangium spinulatum (Roewer)（ゴホントゲザトウムシ), Phalangium tricolor Kulczyński（サンショクザトウムシ), P. bidentatum Kulczyński, Opilio Kishidai Saitô (キシダザトウムシ), O. quadridentatus Wang, Opilio tricolor var. modestus Schenkel = Homolophus serrulatus (Karsch), Opilio hexa-spinulatus Saitô と Oliio koreaus Charitonov = Bidentolophus bidens (Simon), Opilio birmanicus Roewer = Bidentolophus adungius (Roewer), Opilio sunuitensis Nakatsudi（ソニットザトウムシ）とOpilio nipponensis Roewer = Homolophus arcticus Banks（ウデブトザト ウムシ), Euphalangium trinkleri Roewer と Opilio insolitus Roewer = Homolophus tibetanus (Roewer). Phalangium coronatum Redikorzev, ¿ Opilio redikorzevi Roewer = Opilio lederi Roewer, Opilio turcicus Roewer = Opilio saxatilis C. L. Koch, Opilio reginae 